TITLE:

\title{
Changes in visual acuity and foveal photoreceptor integrity in eyes with chronic cystoid macular edema associated with retinal vein occlusion.
}

\section{AUTHOR(S):}

Kurashige, Yumiko; Tsujikawa, Akitaka; Murakami, Tomoaki; Miyamoto, Kazuaki; Ogino, Ken; Muraoka, Yuki; Yoshimura, Nagahisa

\section{CITATION:}

Kurashige, Yumiko ... [et al]. Changes in visual acuity and foveal photoreceptor integrity in eyes with chronic cystoid macular edema associated with retinal vein occlusion.. Retina 2012, 32(4): 792-798

\section{ISSUE DATE:}

2012-04

URL:

http://hdl.handle.net/2433/184456

\section{RIGHT:}

(c) The Ophthalmic Communications Society, Inc.; This is not the

published version. Please cite only the published version.; この論文は 出版社版でありません。引用の際には出版社版をご確認ご利用くださ い。 


\section{CHANGES IN VISUAL ACUITY AND FOVEAL} PHOTORECEPTOR INTEGRITY IN EYES WITH CHRONIC CYSTOID MACULAR EDEMA ASSOCIATED WITH RETINAL 5 VEIN OCCLUSION

Abbreviated title: Chronic CME Associated With RVO

YUMIKO KURASHIGE, MD, AKITAKA TSUJIKAWA, MD, TOMOAKI MURAKAMI, MD, KAZUAKI MIYAMOTO, MD, KEN OGINO, MD, YUKI MURAOKA, MD, NAGAHISA YOSHIMURA, MD

From the Department of Ophthalmology and Visual Sciences, Kyoto University Graduate School of Medicine, Kyoto, Japan.

The authors have no financial interest in the materials presented.

Reprint requests: Akitaka Tsujikawa, MD, Department of Ophthalmology and Visual Sciences, Kyoto University Graduate School of Medicine, Sakyo-ku, Kyoto 606-8507, Japan. Fax: +81-75-752-0933; e-mail: tujikawa@kuhp.kyoto-u.ac.jp 
Key words: cystoid macular edema, optical coherence tomography, photoreceptor layer, retinal vein occlusion.

5

\section{Brief Summary Statement}

Eyes with chronic cystoid macular edema from retinal vein occlusion seem to be at risk of further visual impairment. However, even if a large cystoid space is located in the fovea, its effect on deterioration of the underlying foveal photoreceptor cells is not 10 as strong as is generally expected. 


\section{Structured Abstract}

Purpose: To investigate the change in integrity and thickness of the foveal outer retina in eyes with chronic cystoid macula edema (CME) associated with retinal 5 vein occlusion (RVO)

Methods: We reviewed retrospectively the medical records of 30 eyes of 30 patients who had chronic CME associated with RVO and who had a follow-up of more than 12 months without any treatment. Using optical coherence tomography, we assessed thickness of the outer retina and status of the junction between the inner and outer segments of the photoreceptors (IS/OS) and status of the external limiting membrane (ELM) in the fovea.

Results: Visual acuity (VA) showed a correlation with thickness of the foveal outer retina $(\underline{R=-0.37})$ and with detection of an IS/OS line $(\underline{R=0.65})$ and an ELM line $(\underline{R=0.75})$ beneath the fovea. During the observation period (17.2 \pm 5.5 months), neither VA nor condition of the foveal outer retina showed a significant change, and in no eye were previously detectable lines of IS/OS and ELM just beneath the foveal cystoid space undetectable.

Conclusions: Even if a large cystoid space is located in the fovea, its effect on deterioration of the underlying foveal photoreceptor cells is not as strong as is generally thought. 
Cystoid macula edema (CME) is just one of the many vision-threatening complications associated with retinal vein occlusion (RVO). ${ }^{1-3}$ To date, various treatments have been reported to reduce macular edema from RVO, including grid laser photocoagulation, ${ }^{4}$ pars plana vitrectomy combined with internal limiting 5 membrane peeling, ${ }^{5}$ and intravitreal injection of triamcinolone acetonide, ${ }^{6-9}$ tissue plasminogen activator, ${ }^{10}$ bevacizumab, or ranibizumab, ${ }^{11,12}$ but, in spite of these treatments, some patients have persistent or recurrent CME, and never achieve full visual recovery.

However, we sometimes see patients who maintain good visual acuity (VA) in spite of the chronic CME associated with RVO, and it remains unclear whether or not such eyes should be treated intensively until the CME has regressed completely. With the accumulating number of recent reports on the use of optical coherence tomography (OCT), integrity of the foveal outer retina, especially its outer aspect, is now known to be essential to central visual function. ${ }^{13-16}$ Recently, Ota et al reported that, even in eyes with a large foveal cystoid space associated with branch retinal vein occlusion (BRVO), if the foveal outer photoreceptor layer beneath the cystoid space is intact, VA can be preserved. ${ }^{17}$ However, those authors made no suggestion as to whether or not ophthalmologists should provide additional treatment to such eyes.

To date, little information is available on the natural course of eyes with persistent CME regarding integrity of the foveal photoreceptor layer or VA. In the study reported herein, we reviewed longitudinally the OCT images of eyes with chronic CME associated with RVO and evaluated changes in the foveal outer retina beneath the chronic cystoid space. By assessment of the foveal integrity of these eyes, we suggest that observation with no additional treatment may be possible in eyes with chronic CME, especially if the VA remains good. 


\section{Patients and Methods}

For this retrospective study, we reviewed the medical records of 30 eyes of 30 patients who had chronic CME associated with RVO, and who had follow-up

5 examinations for this chronic CME at Kyoto University Hospital between March 2006 and December 2008; all patients were followed-up for more than 12 months without any treatment. A total of 300 eyes of 287 patients with RVO had been examined; 186 had BRVO, 25 had hemi central retinal vein occlusion (CRVO), and 89 eyes had CRVO. Although most eyes had previously had some type of surgical or medical treatment, eight eyes with chronic CME were excluded from the current study due to additional treatment for their chronic CME. This study was approved by the Institutional Review Board at Kyoto University Graduate School of Medicine and adhered to the tenets of the Declaration of Helsinki.

At the initial visit, all eyes showed characteristic retinal hemorrhage associated with acute RVO and macular edema, in which the total foveal thickness was more than $250 \mu \mathrm{m}$. Most eyes had already undergone surgical or medical treatment for the macular edema, but all eyes showed a persistent large cystoid space beneath the fovea. In the current study, the baseline time point (more than six months after the initial visit) was defined as the follow-up visit when a chronic cystoid space was visible beneath the fovea but retinal hemorrhage was mostly absorbed. Only eyes with a large foveal cystoid space, eyes in which total foveal thickness was $250 \mu \mathrm{m}$ or more at baseline, were included in the current study. Eyes were scheduled for follow-up examinations for the chronic CME after more than 12 months without any treatment; eyes that received any treatment during the observation period were excluded from the current study. Eyes with co-existing ocular disease (i.e., epiretinal membrane, glaucoma, proliferative diabetic retinopathy, or senile cataract that resulted in poor quality OCT images) were also excluded from the study described herein. Of the 30 eyes included in the present study, nine were 
pseudophakic at baseline examination and no eye underwent cataract surgery during the study period.

Twenty-five eyes in this study had undergone treatment for their macular edema before baseline: five eyes were treated with grid laser photocoagulation, two eyes had

5 undergone pars plana vitrectomy, four eyes received posterior sub-Tenon injections of triamcinolone acetonide, and 18 eyes were treated with intravitreal injections of bevacizumab. Scatter laser photocoagulation to the non-perfusion retinal area had been performed in nine eyes. Five eyes had not undergone any treatment. The mean duration of symptoms until the baseline examination was $33.6 \pm 24.5$ months, and the mean duration of observation from baseline was $17.2 \pm 5.5$ months (range, 12-34 months).

At the initial visit of each patient, a comprehensive medical interview was conducted; this included questions concerning systemic diseases such as hypertension, diabetes mellitus, and questions about smoking. Each patient also underwent a complete ophthalmologic examination, including best-corrected VA measurement (Landolt C ring test), slit-lamp biomicroscopy, indirect fundus ophthalmoscopy, fluorescein angiography, and spectral-domain OCT examination. At each follow-up visit, we performed measurement of best-corrected VA, slit-lamp biomicroscopy, indirect fundus ophthalmoscopy, and OCT examinations. Fluorescein angiography was performed if necessary.

In the current study, the macular region was examined by spectral-domain OCT (Spectralis HRA+OCT, Heidelberg Engineering, Heidelberg, Germany; Cirrus HD-OCT, Carl Zeiss, Dublin, California; RS-3000, NIDEK, Gamagori, Japan). On these images, all eyes showed cystoid spaces in the fovea with surrounding, albeit 25 minimal, retinal hemorrhage. Using vertical cross-section OCT scans centered on the fovea, we performed four measurements: total foveal thickness, thickness of the foveal outer retina, thickness of the foveal cystoid space, and transverse length of the foveal cystoid space (Figure1). Total foveal thickness was defined as the distance 
between the vitreoretinal interface and the retinal pigment epithelium in the fovea. Thickness of the foveal outer retina was defined as distance between the posterior surface of the cystoid space and retinal pigment epithelium in the fovea. Transverse length and thickness of the foveal cystoid space were also determined using the 5 vertical cross-section OCT scans.

Furthermore, we examined also the external limiting membrane (ELM) and the junction between the inner and outer segments of the photoreceptors (IS/OS) beneath the fovea. These evaluations were based on gray-scale OCT images obtained in a masked fashion. Each eye was classified by whether or not an IS/OS line and an ELM line in the fovea was detected on the OCT images.

Statistical analysis was performed using software designed for this purpose (StatView, version 5.0; SAS Institute, Cary, North Carolina). All values are presented as mean \pm standard deviation. Best-corrected VA was converted to a logarithm of the minimum angle of resolution (logMAR) equivalent for statistical analysis. For each patient, VA was considered to be changed when the change (in logMAR) was greater than 0.3. Bivariate relationships were analyzed using the Pearson's correlation coefficient. Analysis for two groups was compared using the Student's $t$ test. A $P$ value $<0.05$ was considered to be statistically significant. 


\section{Results}

In the current study, 30 eyes of 30 patients ( 9 men and 21 women) with RVO, ranging in age from 56 to 84 years $(69.0 \pm 12.1$ years $)$, were examined. Of these 30

5 eyes, 20 had BRVO, two had hemi CRVO and eight had CRVO. Table 1 shows characteristics of the patients included in this study. At the initial visit, all eyes showed retinal hemorrhage associated with RVO with macular edema. Mean VA $(\log M A R)$ at the initial visit was $0.39 \pm 0.42$. Mean total foveal thickness at initial visit was $511.4 \pm 185.6 \mu \mathrm{m}$. Of the 30 eyes, 25 (83.3 \%) had undergone prior treatment of the macular edema.

At baseline, most eyes showed substantial absorption of the retinal hemorrhage, but OCT scans showed the foveal cystoid space to be clearly delineated in all eyes. Typically, a large foveal cystoid space was surrounded by parafoveal small spaces, which were located primarily in the external plexiform layer. At the baseline examination, the mean VA was $0.41 \pm 0.32$, and mean total foveal thickness was $507.0 \pm 114.8 \mu \mathrm{m}$. The size of the foveal cystoid spaces varied from 178 to $696 \mu \mathrm{m}$ (mean height, $369.4 \pm 114.3 \mu \mathrm{m}$ ). Beneath these foveal cystoid spaces, the underlying foveal outer retina was detected as distinct layers of various thicknesses, measuring from 32 to $147 \mu \mathrm{m}$ (mean, $83.1 \pm 28.7 \mu \mathrm{m})$. Furthermore, to assess

20 integrity of the foveal outer retina, we examined the IS/OS line and the ELM line. Of the 30 eyes, distinct reflective lines derived from the IS/OS and from the ELM just beneath the foveal cystoid spaces were seen in 19 eyes (63.3\%) and 18 eyes (60.0\%), respectively.

Table 2 shows correlations of baseline VA to baseline OCT measurements and

25 to duration from the initial symptoms. Baseline VA showed no correlation with total foveal thickness at baseline $(\underline{R=0.25, P=0.18})$, but was correlated with thickness of the foveal outer retina $(\underline{\mathrm{R}=-0.37, P=0.047})$ (Figure 2). Moreover, baseline VA was closely correlated with detection of an IS/OS line $(\underline{\mathrm{R}=0.65}, P<0.0001)$ and an ELM 
line $(\underline{R=0.75}, P<0.0001)$ just beneath the foveal cystoid space.

In the current study, the mean observation period for chronic CME with no treatment was $17.2 \pm 5.5$ months (range, $12-34$ months). During this period, mean VA did not show any changes $(0.42 \pm 0.32$ at baseline, and $0.36 \pm 0.34$ at final

5 examination, $\underline{P=0.075}$, Table 3). Of all 30 eyes, two (6.7\%) showed improvement of $\underline{V A}(<-0.3$ in logMAR) and only one $(3.3 \%)$ showed deterioration of $\mathrm{VA}(>0.3$ in logMAR). At the final examination, all eyes continued to show a persistent large cystoid space in the fovea. The total foveal thickness showed no change (507.0 \pm $114.8 \mu \mathrm{m}$ at baseline, and $472.1 \pm 135.1 \mu \mathrm{m}$ at final examination, $\underline{P=0.10}$ ). During the observation period, the foveal cystoid space became somewhat flat $(\underline{P=0.041}$ in thickness, and $\underline{P=0.040}$ in transverse length), but the underlying foveal photoreceptor layer seemed to be well preserved, there being no decrease in thickness of the foveal outer retina $(\underline{P=0.12})$. In addition, integrity of the outer retina beneath the fovea showed no change. At the final visit, distinct reflective lines that were derived from the IS/OS and from the ELM just beneath the foveal cystoid space

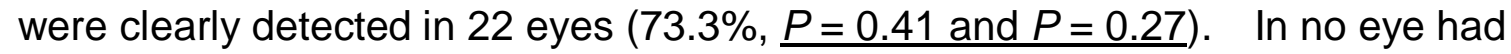
previously detected lines of either IS/OS or ELM become undetectable at the conclusion of the observation period (Figure 3). 


\section{Discussion}

Macular edema is accompanied often by a visual disturbance, depending on the extent of the increased foveal thickness. Recently, however, integrity of the foveal

5 photoreceptor layer, especially its outer aspect, has been suggested to be pivotal to central visual function. ${ }^{13-16}$ Recent reports have indicated that an IS/OS line is a useful hallmark of integrity of the outer photoreceptor layer in various diseases, including central serous chorioretinopathy, ${ }^{18}$ retinitis pigmentosa, ${ }^{19}$ Stargardt's disease ${ }^{20}$ and following surgery for an epiretinal membrane ${ }^{21}$ or a macular hole. ${ }^{22}$ In eyes with resolved macular edema associated with BRVO, Murakami et al suggested that, to achieve good visual recovery, a simple reduction in foveal thickness is insufficient, and that restoration of the structure of the foveal photoreceptors to a more physiologic condition is needed. ${ }^{23}$

In spite of various treatments for the macular edema from RVO, some eyes show persistent or recurrent CME and prolonged visual disturbance. Other patients, however, have good VA despite the presence of a large cystoid space beneath the fovea. In the current study, VA in our patients varied from 20/250 to 20/20, and ten eyes (33\%) of our patients had VA of better than $20 / 30$ at baseline, even though foveal thickness was greater than $250 \mu \mathrm{m}$ and a large foveal cystoid space remained. Under conditions of chronic CME, although VA showed no correlation with total foveal thickness, it was correlated with status of the foveal outer retina. VA showed a correlation with both thickness of the foveal outer retina $(\underline{R=-0.37})$ and with the detection of an IS/OS line $(\underline{R=0.65})$ or an ELM line $(\underline{R=0.75})$ just beneath the foveal cystoid space, so the condition of the underlying foveal outer retina may explain the difference in VA among eyes with chronic CME.

This finding is consistent with the previous report by Ota et al. ${ }^{17}$ Those authors, however, used time-domain OCT, whose resolution was relatively low, and more recent spectral-domain OCT allows more precise evaluation of the retina, including 
condition of the ELM. In addition, because those authors made only a cross-sectional evaluation of chronic CME, no information is available on the change in foveal structures in the presence of chronic CME. In our current study, during the mean observation period of $17.2 \pm 5.5$ months, neither total foveal thickness nor VA

5 showed any significant change. The underlying foveal outer retina appeared well preserved; there was no decrease in the thickness of the foveal outer retina and no change detected in its integrity with the use of IS/OS and ELM. In addition, in no eye in which the lines of IS/OS and ELM just beneath the foveal cystoid spaces were detected were these lines undetectable during the follow-up period. Based on the current findings, although CME appears to carry the risk of further visual impairment, its effect on deterioration of the underlying foveal photoreceptor cells is not as strong as generally thought. Observation with no additional treatment may be possible for chronic CME from RVO, as long as VA is maintained, especially good VA.

There are some limitations to the current study. One limitation is that we evaluated only eyes that had not undergone treatment for the chronic CME for at least 12 months. Throughout this follow-up period, $97 \%$ of our patients maintained baseline VA. Because this is a retrospective study, some eyes were excluded because of additional treatment performed at the request of the patient, especially when subjective symptoms became worse. It should be kept in mind that the current study consisted primarily of eyes that maintained baseline VA in spite of the presence of a foveal cystoid space. Various treatments have recently been reported to be effective for the macular edema associated with RVO. In these cases, however, few data are available regarding the role of active treatments, such as intravitreal injection of anti-vascular endothelial growth factor agents. Further prospective studies may more precisely elucidate the long-term changes in the foveal outer retina of eyes having chronic CME associated with RVO.

Another limitation of the current study is that various types of RVO were included and various treatment regimens were used. In addition, another limitation is that we 
used several types of OCT, although all OCT devices used were of the spectral-domain type. Although in most cases we used Spectralis HRA+OCT, some measurements were made with Cirrus HD-OCT or with RS-3000. It has been reported that Spectralis HRA+OCT demonstrates higher values in automated central

5 macular thickness than do other types of spectral-domain OCT. ${ }^{24}$ To minimize this type of error, all measurements in the current study were made manually with calipers, but we do not think that all errors were eliminated. However, our findings do suggest that, even if chronic CME remains in spite of treatment for the macular edema associated with RVO, relatively good visual function can be maintained if the foveal outer retina (especially its outer aspect) is well preserved. In addition, we suggest that observation without additional treatment is a feasible option for chronic CME, as long as the affected eye maintains good VA. 


\section{References}

1. The Branch Vein Occlusion Study Group. Argon laser photocoagulation for macular edema in branch vein occlusion. Am J Ophthalmol 1984;98:271-282.

2. Glacet-Bernard A, Coscas G, Chabanel A, et al. Prognostic factors for retinal vein occlusion: prospective study of 175 cases. Ophthalmology 1996;103:551-560.

3. Scott IU, VanVeldhuisen PC, Oden NL, et al. SCORE Study report 1: baseline associations between central retinal thickness and visual acuity in patients with retinal vein occlusion. Ophthalmology 2009;116:504-512.

4. The Central Vein Occlusion Study Group. Evaluation of grid pattern photocoagulation for macular edema in central vein occlusion. The Central Vein Occlusion Study Group M report. Ophthalmology 1995;102:1425-1433.

5. Mandelcorn MS, Nrusimhadevara RK. Internal limiting membrane peeling for decompression of macular edema in retinal vein occlusion: a report of 14 cases. Retina 2004;24:348-355.

6. Cekiç $\mathrm{O}$, Chang S, Tseng JJ, et al. Intravitreal triamcinolone injection for treatment of macular edema secondary to branch retinal vein occlusion. Retina 2005;25:851-855.

7. Chen SD, Sundaram V, Lochhead J, Patel CK. Intravitreal triamcinolone for the treatment of ischemic macular edema associated with branch retinal vein occlusion. Am J Ophthalmol 2006;141:876-883.

8. Karacorlu M, Ozdemir H, Karacorlu SA. Resolution of serous macular detachment after intravitreal triamcinolone acetonide treatment of patients with branch retinal vein occlusion. Retina 2005;25:856-860.

9. Tsujikawa A, Fujihara M, Iwawaki T, et al. Triamcinolone acetonide with vitrectomy for treatment of macular edema associated with branch retinal vein occlusion. Retina 2005;25:861-867. 
10. Ghazi NG, Noureddine B, Haddad RS, et al. Intravitreal tissue plasminogen activator in the management of central retinal vein occlusion. Retina 2003;23:780-784.

11. Iturralde D, Spaide RF, Meyerle CB, et al. Intravitreal bevacizumab (Avastin) treatment of macular edema in central retinal vein occlusion: a short-term study. Retina 2006;26:279-284.

12. Pai SA, Shetty R, Vijayan PB, et al. Clinical, anatomic, and electrophysiologic evaluation following intravitreal bevacizumab for macular edema in retinal vein occlusion. Am J Ophthalmol 2007;143:601-606.

13. Otani T, Kishi S, Maruyama Y. Patterns of diabetic macular edema with optical coherence tomography. Am J Ophthalmol 1999;127:688-693.

14. Sakamoto A, Tsujikawa A, Ota M, et al. Evaluation of potential visual acuity in eyes with macular oedema secondary to retinal vein occlusion. Clin Experiment Ophthalmol 2009;37:208-216.

15. Yamaike N, Tsujikawa A, Ota M, et al. Three-dimensional imaging of cystoid macular edema in retinal vein occlusion. Ophthalmology 2008;115:355-362.

16. Ota M, Tsujikawa A, Kita M, et al. Integrity of foveal photoreceptor layer in central retinal vein occlusion. Retina 2008;28:1502-1508.

17. Ota M, Tsujikawa A, Murakami T, et al. Foveal photoreceptor layer in eyes with persistent cystoid macular edema associated with branch retinal vein occlusion. Am J Ophthalmol 2008;145:273-280.

18. Ojima $Y$, Hangai M, Sasahara M, et al. Three-dimensional imaging of the foveal photoreceptor layer in central serous chorioretinopathy using high-speed optical coherence tomography. Ophthalmology 2007;114:2197-2207.

19. Oishi A, Nakamura H, Tatsumi I, et al. Optical coherence tomographic pattern and focal electroretinogram in patients with retinitis pigmentosa. Eye (Lond) 2009;23:299-303.

20. Ergun $\mathrm{E}$, Hermann B, Wirtitsch M, et al. Assessment of central visual function in 
Stargardt's disease/fundus flavimaculatus with ultrahigh-resolution optical coherence tomography. Invest Ophthalmol Vis Sci 2005;46:310-316.

21. Suh MH, Seo JM, Park KH, Yu HG. Associations between macular findings by optical coherence tomography and visual outcomes after epiretinal membrane removal. Am J Ophthalmol 2009;147:473-480.

22. Kang SW, Lim JW, Chung SE, Yi CH. Outer foveolar defect after surgery for idiopathic macular hole. Am J Ophthalmol 2010;150:551-557.

23. Murakami T, Tsujikawa A, Ohta M, et al. Photoreceptor status after resolved macular edema in branch retinal vein occlusion treated with tissue plasminogen activator. Am J Ophthalmol 2007;143:171-173.

24. Mylonas G, Ahlers C, Malamos P, et al. Comparison of retinal thickness measurements and segmentation performance of four different spectral and time domain OCT devices in neovascular age-related macular degeneration. $\mathrm{Br} \mathrm{J}$ Ophthalmol 2009;93:1453-1460. 


\section{Figure legends}

Figure 1. A, Cross-section image of the fovea obtained with optical coherence tomography (OCT) in an eye with chronic cystoid macular edema associated with

5 branch retinal vein occlusion. A large cystoid space is seen in the fovea. The junction between inner and outer segments of the photoreceptors (IS/OS) and the external limiting membrane (ELM) is seen clearly beneath the fovea. B, Magnified image in the region of the foveal cystoid space. Four measurements were obtained from the OCT image. ELM, external limiting membrane; IS/OS, junction between inner and outer segments of the photoreceptors.

Figure 2. Correlation of visual acuity with total foveal thickness $(\mathbf{A})$, thickness of the foveal cystoid space (B), transverse length of the foveal cystoid space (C), and thickness of the foveal outer retina (D) in eyes with chronic cystoid macular edema associated with branch retinal vein occlusion. These four measurements were obtained from sectional images of vertical scans of the fovea obtained with optical coherence tomography at the baseline examination. Visual acuity is converted to logarithm of the minimum angle of resolution (logMAR). Visual acuity shows a correlation with thickness of the foveal outer retina $(\underline{R}=-0.37, P=0.047)$ and with thickness of the foveal cystoid space $(\underline{R=0.41, P=0.025)}$. Closed diamond indicates an eye with the line of junction between inner and outer segments of the photoreceptors (IS/OS) beneath the foveal cystoid space; open diamond indicates an eye without an IS/OS line beneath the foveal cystoid space. Blue frame indicates an eye with a line representing the external limiting membrane (ELM) beneath the foveal cystoid space; red frame indicates an eye without an ELM line beneath the foveal cystoid space.

Figure 3. A 71-year-old woman with branch retinal vein occlusion (BRVO) visited 
our clinic with a 2-week history of decreased visual acuity in the right eye. Visual acuity was 20/22 at the initial visit. A, Fundus photograph shows retinal hemorrhage associated with BRVO. B, Fluorescein angiography shows fluorescein leakage. C, At the initial visit, sectional images along the white line obtained with optical

5 coherence tomography (OCT) show macular edema with foveal cystoid spaces. Total foveal thickness at the initial visit was $470 \mu \mathrm{m}$. D, At baseline (24 months after the initial visit), OCT image shows a large foveal cystoid space. The underlying foveal outer retina seems well preserved (20/30, OD), and thickness of the foveal outer retina is $100 \mu \mathrm{m}$. The line of junction between inner and outer segments of the photoreceptors and the line of the external limiting membrane are seen beneath the foveal cystoid space. E, At 10 months after baseline, OCT image continues to show the large foveal cystoid space. F, At the final visit (16 months after baseline), the size of the foveal cystoid space is somewhat decreased. The thickness and integrity of the underlying foveal outer retina is still well preserved $(20 / 25, O D)$, and thickness of the foveal outer retina is $108 \mu \mathrm{m}$. 
A

transverse length of foveal cyctoid space

B

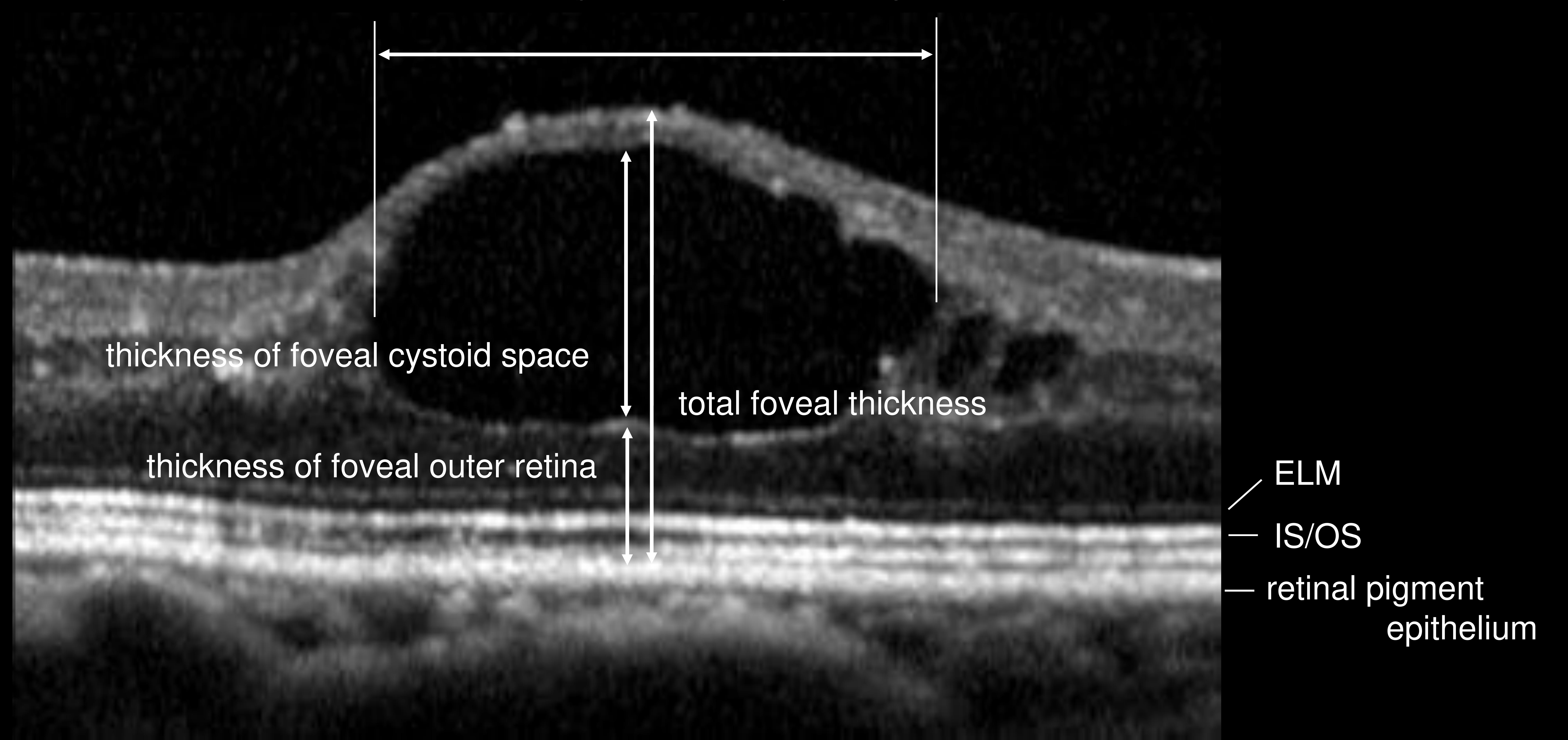



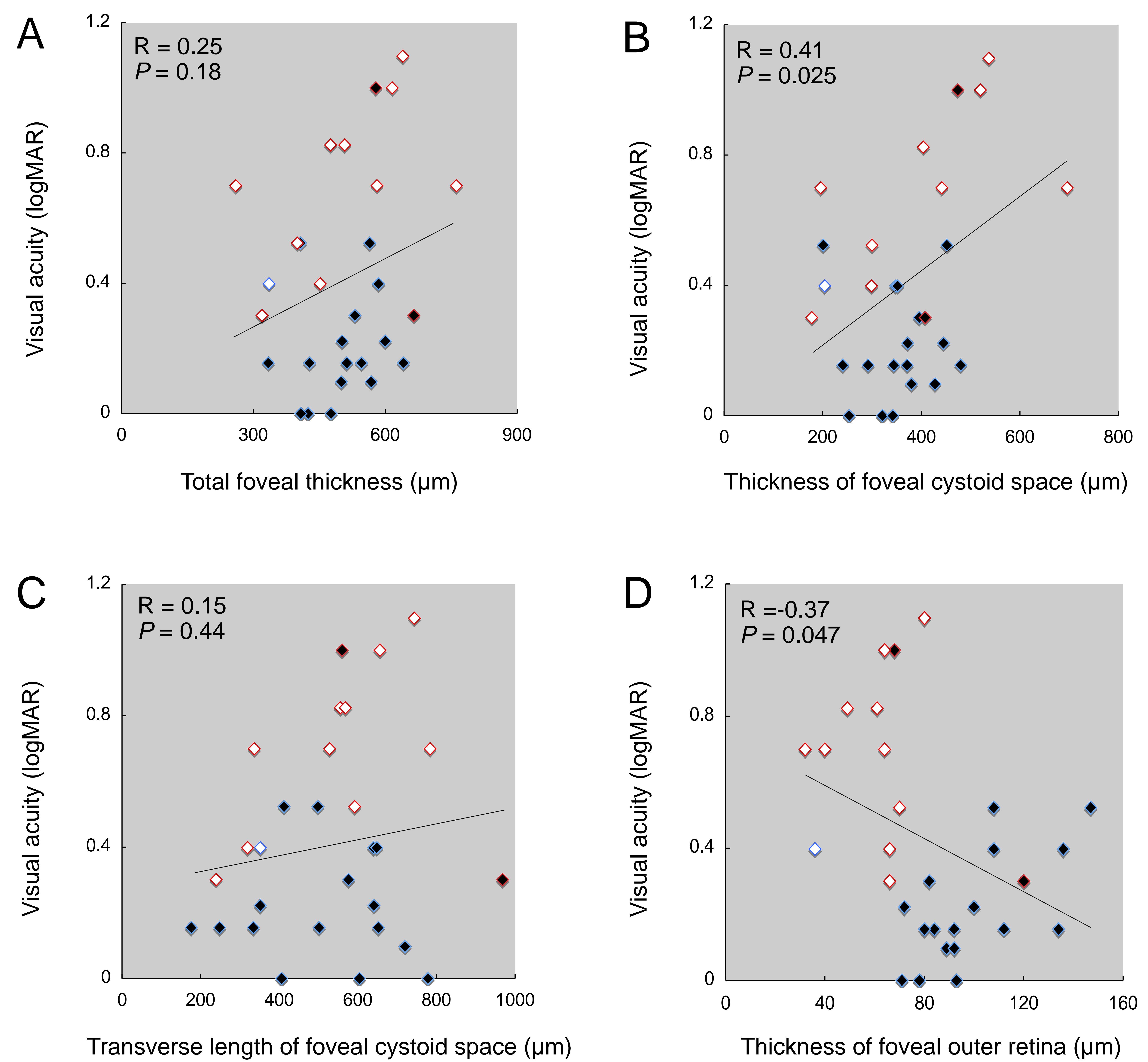

Transverse length of foveal cystoid space $(\mu \mathrm{m})$ 
Table 1. General Characteristics of Study Population

Number of eyes

Age (years)

$69.0 \pm 7.2$

Gender (male/female)

BRVO/hemi-CRVO/CRVO

Systemic hypertension

$16(53.3 \%)$

Diabetes mellitus

Initial examination

Visual acuity (logMAR)

$0.39 \pm 0.42$

Total foveal thickness $(\mu \mathrm{m})$

$511.4 \pm 185.6$

Detection of SRD beneath the fovea

$9(30 \%)$

Detection of IS/OS beneath the fovea

$11(36.7 \%)$

Detection of ELM beneath the fovea

$5(16.6 \%)$

Ischemic/non-ischemic type seen on fluorescein angiography*

Detection of CME beneath the fovea

$26(86.7 \%)$

Duration of symptoms until baseline examination (months)

Baseline examination

Visual acuity (logMAR)

$0.41 \pm 0.32$

Total foveal thickness $(\mu \mathrm{m})$

$507.0 \pm 114.8$

Duration of observation with no treatment (months)

$17.2 \pm 5.5$

Final examination

Visual acuity (logMAR)

$0.36 \pm 0.34$

Total foveal thickness $(\mu \mathrm{m})$

$472.1 \pm 135.0$

Duration of total follow-up (months)

$45.93 \pm 3.72$

BRVO, branch retinal vein occlusion; CRVO, central retinal vein occlusion; logMAR, logarithm of the minimum angle of resolution; SRD, serous retinal detachment; IS/OS, junction between inner and outer segments of the photoreceptors; ELM, external limiting membrane. *Fluorescein angiography was not performed in four patients.

Age, visual acuity, total foveal thickness, duration of symptoms until baseline examination, duration of observation with no treatment, and duration of total follow-up are shown as mean \pm standard deviation. 
Table 2. Associations of Baseline Visual Acuity with Other Baseline Measurements with Optical

Coherence Tomography in Eyes with Chronic Cystoid Macular Edema Associated with Retinal Vein

Occlusion

\begin{tabular}{lcc}
\hline & $r$ & $P$ value \\
\hline Total retinal thickness $(\mu \mathrm{m})$ & 0.25 & 0.18 \\
Thickness of foveal cystoid space $(\mu \mathrm{m})$ & 0.41 & 0.025 \\
Transverse length of foveal cystoid space $(\mu \mathrm{m})$ & 0.15 & 0.44 \\
Thickness of foveal outer retina $(\mu \mathrm{m})$ & -0.37 & 0.047 \\
Detection of IS/OS beneath the fovea & 0.65 & $<0.0001$ \\
Detection of ELM beneath the fovea & 0.75 & 0.0001 \\
Duration from the initial symptoms (months) & 0.31 & 0.097 \\
\hline
\end{tabular}

logMAR, logarithm of the minimum angle of resolution; IS/OS, junction between inner and outer segments of the photoreceptors; ELM, external limiting membrane. 
Table 3. Changes in Visual Acuity and Optical Coherence Tomography Measurements between Baseline and Final Examination

\begin{tabular}{lrrc}
\hline & Baseline & $\begin{array}{c}\text { Final } \\
\text { Examination }\end{array}$ & $\begin{array}{c}P \\
\text { Value }\end{array}$ \\
\hline Visual acuity (logMAR) & $0.41 \pm 0.32$ & $0.36 \pm 0.34$ & 0.075 \\
Total foveal thickness $(\mu \mathrm{m})$ & $507.0 \pm 114.8$ & $472.1 \pm 135.1$ & 0.10 \\
Thickness of foveal outer retina $(\mu \mathrm{m})$ & $83.1 \pm 28.7$ & $91.3 \pm 19.8$ & 0.12 \\
Thickness of foveal cystoid space $(\mu \mathrm{m})$ & $369.4 \pm 114.3$ & $322.0 \pm 152.2$ & 0.041 \\
Transverse length of foveal cystoid space $(\mu \mathrm{m})$ & $536.9 \pm 187.8$ & $629.2 \pm 225.8$ & 0.040 \\
Detection of IS/OS beneath the fovea $(\%)$ & 63.3 & 73.7 & 0.41 \\
Detection of ELM beneath the fovea $(\%)$ & 60.0 & 73.7 & 0.27 \\
\hline
\end{tabular}
logMAR, logarithm of the minimum angle of resolution; IS/OS, junction between inner and outer segments of the photoreceptors; ELM, external limiting membrane.

Visual acuity, total foveal thickness, thickness of the foveal photoreceptor layer, thickness of the foveal cystoid space, and transverse length of the foveal cystoid space are shown as mean \pm standard deviation. 